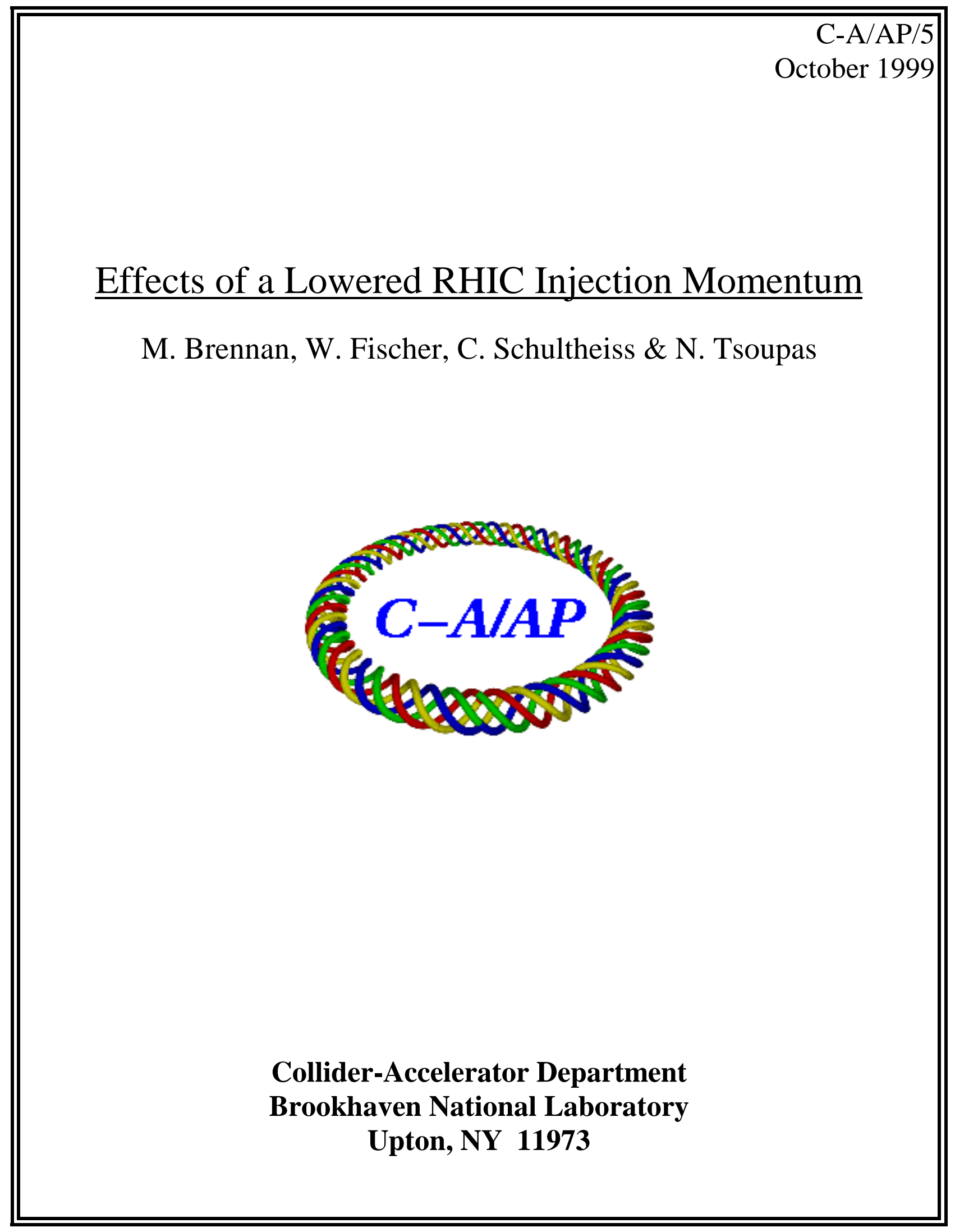




\title{
Effects of a Lowered RHIC Injection Momentum
}

\author{
M. Brennan, W. Fischer, C. Schultheiss and N. Tsoupas
}

October 27, 1999

\begin{abstract}
During the RHIC commissioning run with gold ions the injection kickers were about $15 \%$ too weak. One way to increase the effective kick strength is to lower the injection momentum. In this note the effects of a lower injection momentum are investigated. We consider the AGS extraction performance, the transverse matching, the beam size, the longitudinal matching, nonlinear magnetic field errors and the dynamic aperture, persistent currents, intrabeam scattering, and the power supply regulation. We study cases with injection momenta lowered by up to $30 \%$ compared to the nominal value reported in the design manual [1].
\end{abstract}

\section{Introduction}

During the RHIC commissioning run in summer of 1999 it became apparent that the injection kickers were about $15 \%$ too weak to bring the incoming beam onto a flat vertical closed orbit in the ring. About $5 \%$ of the lacking strength can be accounted for by the rhic99 optics which is different from the nominal one due to lacking power supplies in the interaction regions [2]. During the RHIC Retreat [3] is was proposed to gain $15 \%$ of effective kick strength through

- the correct optics ( $5 \%)$,

- raising the kicker voltage by $5 \%$ and

- lowering the injection momentum by $5 \%$.

In this note the third of these options is explored in some detail. Effects considered are limits in the AGS extraction performance, the transverse matching, the beam size, the longitudinal matching, the change in nonlinear magnetic field errors and the related dynamic aperture change, chromaticity changes through persistent currents, intrabeam scattering, and the power supply regulation at lower excitations levels.

During the commissioning the best measurement for the relativistic $\gamma$ of the injected gold beam came from the rf frequency and the design circumference, resulting in a value of $\gamma=12.09$. Our benchmark case in this note is $\gamma=12$. We study cases with a $\gamma$ lowered by $5 \%, 10 \%, 15 \%, 20 \%$ and $30 \%$. We consider only gold ions here. Except for the longitudinal matching and intrabeam scattering all effects investigated are the similar for gold ions and protons. For protons the limit below which it is undesirable to inject is the transition energy at $\gamma=22.9$. A gold beam with the same rigidity has a relativistic factor of $\gamma=9.3$. 


\section{AGS Extraction and AtR Transfer Line}

We consider the performance of the AGS extraction elements and the transverse matching into the transfer line. We restrict ourselves the case of a RHIC injection momentum lowered by $30 \%$. There are no transverse matching problems when the injection momentum is lowered by only 15\%. The main devices which are part of the FEB extraction process are

1. the back-leg windings of certain AGS magnets,

2. the G10 fast extraction kicker,

3. the H10 extraction septum.

The back-leg windings used in the extraction are shown in Tab. 1. The power-supplies that power the back-leg windings run reliably down to $100 \mathrm{~A}$ [4]. The required current for extraction with a $30 \%$ lower momentum, corresponding to $\gamma=8.4$, would be $450 \mathrm{~A}$ and $900 \mathrm{~A}$ respectively. Beam with a rigidity corresponding to $\gamma=5$ for gold ions has been extracted from the AGS using the back-legged magnets, the G10 extraction kicker and the H10 extraction septum.

Table 1: Back-leg windings, corresponding power supplies and maximum current of certain AGS extraction elements.

\begin{tabular}{|c|c|c|c|c|}
\hline Magnet back-leg winding & $\begin{array}{l}\text { F08,F09, } \\
\text { G02,G03 }\end{array}$ & G16,G17 & $\mathrm{H} 04, \mathrm{H} 05$ & $\begin{array}{c}\mathrm{H} 18, \mathrm{H} 19, \\
\mathrm{I} 12, \mathrm{I} 13\end{array}$ \\
\hline$\overline{P S} n$ & G09A & G09B & H10B & $\mathrm{H} 10 \mathrm{~A}$ \\
\hline Maximum current $I_{\max }[\mathrm{A}]$ & 600 & 1200 & 1200 & 600 \\
\hline
\end{tabular}

Tab. 2 shows four scenarios where the AGS tune and extraction radius have been varied to match the lattice functions $\beta_{x, y}, \alpha_{x, y}$ and the dispersion $\eta_{x, y}$ to the target at H13. The table shows that a relatively good agreement can be achieved. This will keep the optics of the u-line almost the same as the nominal optics. The matching of the u-line to the w-line guarantees identical beam parameters at the rest of the AtR line. The calculations were performed using a modified version of the computer code BEAM which utilizes measured magnetic field maps of the combined function magnets of the AGS [5].

\section{Beam Size}

We compute the physical aperture in units of the transverse rms emittance $\sigma_{x, y}$ at a number of elements in the AtR and RHIC. The $1 \sigma_{x, y}$ betatron amplitude is given by

$$
a_{1 \sigma x, y}=\sqrt{\frac{\epsilon_{x, y}^{N}}{6(\beta \gamma)} \beta_{x, y}}
$$

where $(\beta \gamma)$ are the relativistic factors and $\epsilon_{x, y}^{N}$ is the $95 \%$ normalized emittance. We choose the elements where the physical aperture is most limited. Tab. 3 shows the limitations for a $10 \mathrm{~mm} \cdot \mathrm{mrad}$ beam centered in the beam pipe for different RHIC injection momenta. 
Table 2: Different scenarios for the transverse matching of the AGS extracted beam at element H13. The matching targets are the lattice functions at the nominal extraction momentum.

\begin{tabular}{llccccc}
\hline Case & Unit & Target & $\# 1$ & $\# 2$ & $\# 3$ & $\# 4$ \\
Relativistic $\gamma$ & {$[1]$} & & $8.85+0.25 \%$ & 8.85 & 8.85 & 8.85 \\
\hline$I_{Q_{x}}$ & {$[\mathrm{~A}]$} & & 0 & 0 & 95 & 130 \\
$I_{Q_{y}}$ & {$[\mathrm{~A}]$} & & 0 & 0 & -65 & -25 \\
$Q_{x}$ & {$[1]$} & & 8.67 & 8.72 & 8.75 & 8.78 \\
$Q_{y}$ & {$[1]$} & & 8.77 & 8.77 & 8.78 & 8.75 \\
$\mathrm{G} 10$ bump & {$[\mathrm{mrad}]$} & & 0.91 & 0.96 & 0.93 & 0.91 \\
$\mathrm{H} 10$ bump & {$[\mathrm{mrad}]$} & & 0.84 & 0.92 & 0.92 & 0.92 \\
\hline $\mathrm{H} 13 \mathrm{x}$ & {$[\mathrm{mm}]$} & & 450.9 & 450.9 & 450.9 & 450.9 \\
$\mathrm{H} 13 \mathrm{x}$ & {$[\mathrm{mrad}]$} & & 69.5 & 69.5 & 69.5 & 69.5 \\
$\mathrm{H} 13 \beta_{x}$ & {$[\mathrm{~m}]$} & 37.5 & 33.9 & 33.8 & 32.8 & 32.7 \\
$\mathrm{H} 13 \alpha_{x}$ & {$[1]$} & -4.1 & -3.6 & -3.6 & -3.5 & -3.5 \\
$\mathrm{H} 13 \beta_{y}$ & {$[\mathrm{~m}]$} & 6.5 & 7.5 & 7.5 & 7.7 & 7.6 \\
$\mathrm{H} 13 \alpha_{y}$ & {$[1]$} & 0.8 & 1.1 & 1.1 & 1.1 & 1.1 \\
$\mathrm{H} 13 \eta_{x}$ & {$[\mathrm{~m}]$} & -1.5 & -1.5 & -1.1 & -0.9 & -0.6 \\
$\mathrm{H} 13 \eta_{x}^{\prime}$ & {$[1]$} & -0.1 & -0.1 & -0.1 & -0.0 & -0.0 \\
\hline
\end{tabular}

Table 3: Physical aperture at selected locations in the AtR transfer line and RHIC. Assumed is a beam with a $95 \%$ normalized emittance of $10 \mathrm{~mm} \cdot \mathrm{mrad}$, centered in the beam pipe. Transfer line element names are from the $\mathrm{x}$-line, aperture is abbreviated with "Aper".

\begin{tabular}{|c|c|c|c|c|c|c|c|c|c|c|}
\hline \multirow{2}{*}{\multicolumn{3}{|c|}{$\begin{array}{l}\text { Momentum reduction } \\
\text { Relativistic } \gamma\end{array}$}} & [\%] & - & 5 & 10 & 15 & 20 & 30 & \\
\hline & & & [1] & 12.0 & 11.4 & 10.8 & 10.2 & 9.6 & 8.4 & \\
\hline Location & $\begin{array}{l}\text { Aper } \\
{[\mathrm{mm}]}\end{array}$ & Plane & $\begin{array}{l}\beta_{x, y} \\
{[\mathrm{~m}]}\end{array}$ & $\begin{array}{l}\text { Aper } \\
{\left[\sigma_{x, y}\right]} \\
\end{array}$ & $\begin{array}{l}\text { Aper } \\
{\left[\sigma_{x, y}\right]} \\
\end{array}$ & $\begin{array}{l}\text { Aper } \\
{\left[\sigma_{x, y}\right]} \\
\end{array}$ & $\begin{array}{l}\text { Aper } \\
{\left[\sigma_{x, y}\right]} \\
\end{array}$ & $\begin{array}{l}\text { Aper } \\
{\left[\sigma_{x, y}\right]} \\
\end{array}$ & $\begin{array}{l}\text { Aper } \\
{\left[\sigma_{x, y}\right]} \\
\end{array}$ & Comment \\
\hline \multicolumn{11}{|l|}{ AtR } \\
\hline uq1 & 31.8 & hor & 96 & 8.7 & 8.5 & 8.3 & 8.0 & 7.8 & 7.3 & round \\
\hline uq9 & 50.8 & hor & 176 & 10.3 & 10.0 & 9.7 & 9.5 & 9.2 & 8.6 & round \\
\hline ud4-ud5 & 39.0 & ver & 51 & 14.7 & 14.3 & 13.9 & 13.5 & 13.1 & 12.3 & C-type \\
\hline wd1-wd8 & 39.0 & ver & 55 & 14.1 & 13.8 & 13.4 & 13.0 & 12.6 & 11.8 & C-type \\
\hline wq6 & 50.8 & hor & 105 & 13.3 & 13.0 & 12.6 & 12.3 & 11.9 & 11.1 & round \\
\hline $\mathrm{xd} 1 \ldots 27$ & 32.0 & ver & 27 & 16.5 & 16.1 & 15.7 & 15.2 & 14.8 & 13.8 & B-type \\
\hline xq6 & 50.8 & hor & 99 & 13.7 & 13.4 & 13.0 & 12.6 & 12.3 & 11.5 & round \\
\hline xp1 & 22.0 & ver & 27 & 11.4 & 11.2 & 10.9 & 10.6 & 10.2 & 9.6 & ver. flat \\
\hline xlamb & 22.0 & hor & 23 & 12.3 & 12.0 & 11.7 & 11.3 & 11.0 & 10.3 & hor. flat \\
\hline \multicolumn{11}{|l|}{ RHIC } \\
\hline xki & 20.0 & ver & 40 & 8.5 & 8.3 & 8.0 & 7.8 & 7.6 & 7.1 & round \\
\hline arc quad & 38.0 & hor/ver & 50 & 14.4 & 14.1 & 13.7 & 13.3 & 12.9 & 12.1 & round \\
\hline IR quad & 63.0 & hor/ver & 150 & 13.8 & 13.5 & 13.1 & 12.7 & 12.3 & 11.5 & round \\
\hline
\end{tabular}


Most limiting are the AtR quadrupole $u q 1$ in the horizontal plane and the RHIC injection kicker in the vertical plane. Both have still $7 \sigma$ of physical aperture for an injection momentum lowered by $30 \%$. The above statement is true also when we include the contribution of the dispersion of the AtR line to the beam size.

\section{Longitudinal Matching}

Tab. 4 shows the momentum spread, synchrotron frequency and slipping factor in the AGS for different extraction momenta as well as the rf voltage that is needed in RHIC to match the beam longitudinally. If the momentum is lowered by $20 \%$, corresponding to a relativistic $\gamma$ of 9.6 , there are $700 \mathrm{kV}$ required in RHIC, beyond the current limit of $500 \mathrm{kV}$. If the momentum is lowered by $30 \%$, corresponding to a relativistic $\gamma$ of 8.4 , the extraction momentum in the AGS would be below and close to making the operation of rf loops difficult. The longitudinal matching condition of the gold beam therefore prevents a reduction of the RHIC injection momentum by more than $15 \%$.

Table 4: Momentum spread, synchrotron frequency and slipping factor in the AGS for different extraction momenta together with the matching RF voltage in RHIC.

\begin{tabular}{llcccccc}
\hline Momentum reduction & {$[\%]$} & - & 5 & 10 & 15 & 20 & 30 \\
Relativistic $\gamma$ & {$[1]$} & 12.0 & 11.4 & 10.8 & 10.2 & 9.6 & 8.4 \\
\hline AGS $\Delta p / p$ & {$\left[10^{-3}\right]$} & 1.15 & 1.24 & 1.35 & 1.43 & 1.70 & 3.10 \\
AGS $f_{s}$ & {$[\mathrm{~Hz}]$} & 126 & 122 & 116 & 107 & 93 & 33 \\
AGS $\eta$ & {$[1]$} & 0.0069 & 0.0061 & 0.0053 & 0.0042 & 0.0030 & -0.0003 \\
RHIC $V_{\text {rf }}$ to match & {$[\mathrm{kV}]$} & 186 & 240 & 303 & 404 & 700 & - \\
\hline
\end{tabular}

\section{Nonlinear Magnetic Field Errors and Dynamic Aperture}

We assume here that the nonlinear magnetic field errors are purely of a geometric nature. In this case, the coefficients $\left(b_{n}, a_{n}\right)$ in the field expansion

$$
B_{y}+i B_{x}=B_{0}\left[1+\sum_{n=0}^{\infty}\left(b_{n}+i a_{n}\right)\left(\frac{x+i y}{r_{0}}\right)^{n}\right]
$$

do not change with the main field $B_{0} . r_{0}$ is a reference radius. Other effects that contribute to field errors are saturation and persistent currents. Saturation effects can be neglected at injection energy, the change of chromaticity due to persistent current decay is treated in the next section.

To simplify the discussion we consider only transverse motion and on-momentum par- 
ticles. The Hamiltonian can then be written as $[6]$

$$
H\left(x, z, x^{\prime}, z^{\prime} ; s\right)=\frac{1}{2} x^{\prime 2}+\frac{1}{2} z^{\prime 2}+k_{x}(s) x^{2}-k_{z}(s) z^{2}+\sum_{\substack{m n \\ m, n \geq 1 \\ m+n \geq 2}}^{\infty} \mathfrak{a}_{m n}(s) x^{m} z^{n}
$$

where the $\mathfrak{a}_{m n}$ are given by

$$
\begin{aligned}
& \mathfrak{a}_{m n}=(-1)^{n / 2} \frac{(m+n-1) !}{m ! n !} b_{m+n+1} r_{0}^{-(m+n)}, \text { for } n \text { even ("normal" multipoles), } \\
& \mathfrak{a}_{m n}=(-1)^{(n+1) / 2} \frac{(m+n-1) !}{m ! n !} a_{m+n+1} r_{0}^{-(m+n)}, \text { for } n \text { odd ("skew" multipoles). }
\end{aligned}
$$

$k_{x}(s)$ and $k_{y}(s)$ are measures for the focusing strength (strong and weak). The Hamiltonian (3), and therefore the dynamic aperture, is independent of the particle momentum. However, with a lower momentum one has to accommodate a larger beam. A 30\% reduction in the injection momentum leads to a $15 \%$ larger beam.

In an earlier study [7] 100,000 turn loss borders were found at 10-12 $\sigma$. 100,000 turn chaotic borders, normally a pessimistic estimate of the dynamic aperture, were found at $7-10 \sigma$. The machine studied used protons with a relativistic $\gamma$ of 27 and the nominal tunes of $\left(Q_{x}, Q_{y}\right)=(28.19,19,18)$. The simulation was done in the 6 dimensions and included tune ripple of various strength. We would not expect an insufficient dynamic aperture when the injection momentum is lowered by $30 \%$.

\section{Chromaticity Change Due to Persistent Current Decay}

Once the main magnets of RHIC are ramped to the injection level the persistent currents created will decay with time and change the sextupole component of the magnetic field. This leads to a slow change of the chromaticity. When the acceleration ramp starts the sextupoles will change back to their original value in a short time interval thereby changing the chromaticity rapidly. This effect is known as snap-back.

Bench measurements of the time dependent sextupole field in the dipoles have been made at $660 \mathrm{~A}$. Fig. 1 shows the measurements of 20 RHIC arc dipoles. Typically, the sextuple field changes by 1 unit in 5 minutes. We assume that the persistent currents are the approximately independent of the main current [10] and scale the measurements at $660 \mathrm{~A}$ accordingly to lower values of the dipole current.

We use as definition for the horizontal chromaticity

$$
\xi_{x}=\frac{\Delta Q_{x}}{\Delta p / p}
$$




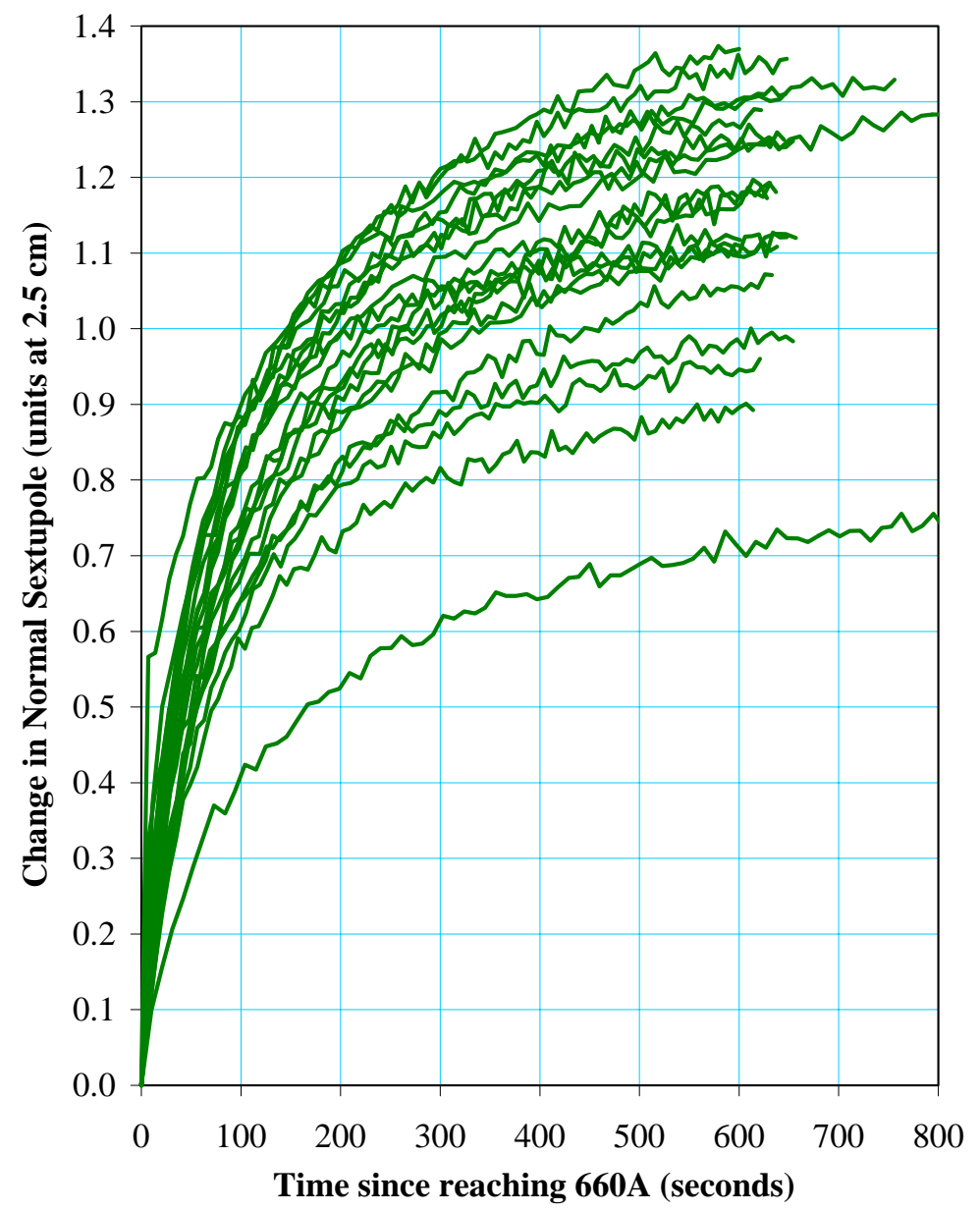

Figure 1: Time-dependent change of the sextupole coefficient in 20 RHIC dipoles in units or $10^{-4}$ at a reference radius of $2.5 \mathrm{~cm}[10]$.

The change of chromaticity $\Delta \xi_{x}$ associated with the change of the sextupole component $b_{2}$ in the main dipole field $B_{0}$ can then be computed as (see for example [8])

$$
\Delta \xi_{x}=-\frac{1}{2 \pi} \frac{1}{(B \rho)} \oint \beta_{x}(s) \frac{B_{0} \Delta b_{2}(s)}{r_{0}^{2}} \eta_{x}(s) \mathrm{d} s .
$$

where $\eta_{x}$ is the dispersion and $r_{0}$ the reference radius from the magnet measurements. $B_{0}$ is the main dipole field in the measurement of the persistent current effects and is different from the $B$ in the rigidity $(B \rho) . \Delta \xi_{x}$ can now be approximated as

$$
\Delta \xi_{x}=-\frac{B_{0}}{B} \frac{\Delta b_{2}}{r_{0}^{2}}\left\langle\beta_{x}(s) \eta_{x}(s)\right\rangle
$$

where the angle $\langle$.$\rangle indicates the average over one arc dipole. Given the RHIC cell length$ $L_{p}=29.6 \mathrm{~m}$, the cell phase advance $\mu=1.41 \mathrm{rad}$ and the cell bending angle $\phi=77.8 \mathrm{mrad}$, 
the average $\langle$.$\rangle in Eq. (8) can be computed, using a thin-lens approximation [9], as$

$$
\left\langle\beta_{x}(s) \eta_{x}(s)\right\rangle=\int_{l_{1}}^{l_{2}} \frac{\mathrm{d} s}{l_{2}-l_{1}}\left[\beta^{+}-\frac{2 s\left(1+\sin \frac{\mu}{2}\right)}{\cos \frac{\mu}{2}}+\frac{4 s^{2} \tan \frac{\mu}{2}}{L_{p}}\right] \cdot\left[\eta^{+}\left(1-\frac{2 s \sin \frac{\mu}{2}}{L_{p}}\right)+\frac{s^{2} \phi}{2 L_{p}}\right]_{(9)}
$$

where the limits $l_{1} \approx 2.5 \mathrm{~m}$ and $l_{2} \approx 12 \mathrm{~m}$ are the $s$-positions of the beginning and end of an arc dipole relative to the center of a cell quadrupole. The maximum beta function $\beta^{+}$ and dispersion $\eta^{+}$of the cell are given by [9]

$$
\beta^{+}=\frac{L_{p}\left(1+\sin \frac{\mu}{2}\right)}{\sin \mu} \text { and } \eta^{+}=\frac{L_{p} \phi\left(1+\frac{1}{2} \sin \frac{\mu}{2}\right.}{4 \sin ^{2} \frac{\mu}{2}} .
$$

We obtain from a numerical integration

$$
\left\langle\beta_{x}(s) \eta_{x}(s)\right\rangle=34.2 \mathrm{~m}^{2} .
$$

Tab. 5 shows the change in chromaticity for the different injection momenta after 5 minutes. With a momentum spread $\Delta p / p$ of about 0.001 off-momentum particles experience a change in the horizontal tune of up to 0.01 within 5 minutes when the injection momentum is lowered by $30 \%$. This should be of some concern.

Table 5: Change in the horizontal chromaticity due to persistent currents for different injection momenta after 5 minutes.

\begin{tabular}{llcccccc}
\hline Momentum reduction & {$[\%]$} & - & 5 & 10 & 15 & 20 & 30 \\
Relativistic $\gamma$ & {$[1]$} & 12.0 & 11.4 & 10.8 & 10.2 & 9.6 & 8.4 \\
\hline$I_{\text {dipole }}$ & {$[\mathrm{A}]$} & 543 & 524 & 489 & 462 & 434 & 380 \\
$\Delta \xi_{x}$ after $5 \mathrm{~min}$ & {$[1]$} & -6.4 & -6.7 & -7.1 & -7.5 & -8.0 & -9.1 \\
\hline
\end{tabular}

\section{$7 \quad$ Intrabeam Scattering}

To investigate the change in the beam size growth times caused by intrabeam scattering we use the model described in Ref. [11]. We assume that the RHIC ring is only made up of regular FODO cells and use the nominal bunch intensity of $10^{9}$ particles. We compute the growth times

$$
\tau_{x, y, s}=\left(\frac{1}{\sigma_{x, y, s}} \frac{\mathrm{d} \sigma_{x, y, s}}{\mathrm{~d} t}\right)^{-1}
$$

for different momenta. The results are shown in Tab. 6. Growth rates scale approximately linearly with $\gamma$ and with intensity.

For the cases under consideration, the initial longitudinal growth rate due to intrabeam scattering will not be reduced by more than $25 \%$ and should not cause additional severe problems. 
Table 6: Initial growth times for a gold beam at nominal intensity with different momenta in RHIC.

\begin{tabular}{llcccccc}
\hline Momentum reduction & {$[\%]$} & - & 5 & 10 & 15 & 20 & 30 \\
Relativistic $\gamma$ & {$[1]$} & 12.0 & 11.4 & 10.8 & 10.2 & 9.6 & 8.4 \\
\hline$\tau_{x}$ & {$[\mathrm{~min}]$} & -38.0 & -33.1 & -28.8 & -25.0 & -21.6 & -16.1 \\
$\tau_{y}$ & {$[\mathrm{~min}]$} & -22.0 & -19.9 & -17.9 & -16.1 & -14.3 & -11.2 \\
$\tau_{s}$ & {$[\mathrm{~min}]$} & 2.2 & 2.1 & 2.0 & 1.9 & 1.8 & 1.6 \\
\hline
\end{tabular}

\section{Power Supply Regulation}

During the commissioning run measurements of the current regulation of the main dipoles at $543 \mathrm{~A}$ showed

- an absolute current error of 30ppm (0.165A),

- a repeatability of better than 5ppm (0.028A),

- a stability of better than $2 \mathrm{ppm}(0.011 \mathrm{~A})$.

Assuming that the set point is adjusted until the required current is realized the maximum current error is less than $0.039 \mathrm{~A}$. This absolute error is independent of the dipole current. The relative current errors at different injection momenta are given in Tab. 7 , they scale inversely proportional with the injection momentum. During commissioning stability could be maintained at currents as low as $50 \mathrm{~A}$.

Table 7: Absolute and relative current error at different excitation levels of the main dipole bus.

\begin{tabular}{llcccccc}
\hline Momentum reduction & {$[\%]$} & - & 5 & 10 & 15 & 20 & 30 \\
Relativistic $\gamma$ & {$[1]$} & 12.0 & 11.4 & 10.8 & 10.2 & 9.6 & 8.4 \\
\hline$I_{\text {dipole }}$ & {$[\mathrm{A}]$} & 543 & 524 & 489 & 462 & 434 & 380 \\
$I_{\text {error }}$, absolute & {$[\mathrm{A}]$} & 0.039 & 0.039 & 0.039 & 0.039 & 0.039 & 0.039 \\
$I_{\text {error }} / I_{\text {dipole }}$ & $\mathrm{ppm}$ & 72 & 76 & 80 & 84 & 90 & 103 \\
\hline
\end{tabular}

\section{Conclusion}

The investigated effects show no operational limit when the RHIC injection momentum is lowered by $15 \%$. A beam with an even lower momentum cannot be matched longitudinally by the RHIC rf. However, all effects are likely to deteriorate the beam lifetime slightly and should be reviewed after more operational experience has been gained. 


\section{Acknowledgments}

The authors are thankful for discussions with numerous people in the Collider-Accelerator Department, in particular L. Ahrens, G. Ganetis, A. Jain, W. MacKay, T. Roser, S. Peggs, S. Tepikian, P. Wanderer and J. Wei. We thank J. Wei for providing a program to compute growth times due to intrabeam scattering and A. Jain for providing magnet measurement data.

\section{References}

[1] "RHIC Design Manual“, revision of April 1998.

[2] N. Tsoupas and S. Tepikian, private communication (1999).

[3] S. Peggs, RHIC Retreat (1999). http://www.cadops.bnl.gov/RHIC/YearZero/retreat/

[4] MCR Logbook FE.307.1.9801 pp. 18-21 (1998).

[5] N. Tsoupas et al., "Closed Orbit Calculations at AGS and Extraction Beam Parameters at H13", BNL AD/RHIC/RD-75 (1994).

[6] W. Fischer, "An Experimental Study on the Long-term Stability of Particle Motion in Hadron Storage Rings", PhD thesis Hamburg University, DESY 95-235 (1995) and CERN SL/96-10 (AP) (1996).

[7] W. Fischer and T. Satogata, "A Simulation Study on Tune Modulation in RHIC", BNL RHIC/AP/109 (1996).

[8] M. Conte, W.W. MacKay, "An Introduction to the Physics of Particle Accelerators", World Scientific (1991).

[9] E. Keil, "Lattices for Collider Storage Rings", in "Handbook of Accelerator Physics and Engineering" edited by A.W. Chao and M. Tigner, World Scientific (1999).

[10] A. Jain, private communication (1999).

[11] J. Wei, "Evolution of Hadron Beams under Intrabeam Scattering", proceedings of the 1993 IEEE Particle Accelerator Conference, Washington, D.C. (1993). 